\title{
LAS INVESTIGACIONES DE LA COMISIÓN INTERAMERICANA PARA LA ENSEÑANZA DE LAS RELACIONES PÚBLICAS
}

\section{INVESTIGATIONS OF THE INTER-AMERICAN TEACHING OF PUBLIC RELATIONS}

\section{AUTOR}

Juan José Ugalde Fernández

Profesor titular del CAP 2 de la Facultad de Ciencias de la Información de la Universidad Complutense. Madrid (España)

jjugalde@hotmail.com

\section{RESUMEN}

La Comisión Interamericana de Relaciones Públicas ha sido la gran impulsora de los estudios de los aspectos técnicos y profesionales de las Relaciones Públicas en los últimos años. De sus estudios, desarrollados por la OIT (Oficina Internacional del Trabajo) se desprenden una serie de conclusiones sobre formas y contenidos propios de las Relaciones Públicas que han pasado a ser tomados ya, pese a su juventud, como canónicos en esta área. El artículo los repasa y extrae conclusiones sobre el I Encuentro Iberoamericano de Profesores de Relaciones Públicas.

\section{PALABRAS CLAVE}

Comisión Interamericana de Relaciones Públicas - Oficina Internacional del Trabajo Profesores de Relaciones Públicas. 


\section{ABSTRACT}

Inter-American Commission on Public Relations has been the great promoter of studies and technical aspects of Public Relations professionals in recent years. From his studies, developed by the ILO (International Labour Office) come from a number of conclusions about form and content specific to public relations that have become taken and, despite his youth, as canonical in this area. The article reviews and draws conclusions on the First Iberoamerican Meeting of Teachers of Public Relations.

\section{KEY WORDS}

Inter-American Public Relations Committee - International Labour Office - Professors in Public Relations.

\section{ÍNDICE}

1. Marco Conceptual para las funciones de las Relaciones Públicas

1.1. Función básica: Asesoría.

1.2. Función básica: Investigación.

1.3. Función básica: Planificación.

1.4. Función básica: Ejecución (Comunicación).

2. Posiciones de diversos autores y profesionales de las Relaciones Públicas en cuanto al perfil de la profesión.

3. $1^{\text {er }}$ Encuentro iberoamericano de profesores de relaciones públicas auspiciado por la confederación iberoamericana de Relaciones Públicas (CIRP)

3.1. Conclusiones del Encuentro:

3.2. Acuerdo final

4. Bibliografía. 


\section{Marco Conceptual para las funciones de las Relaciones Públicas}

Las investigaciones más importantes en el área de las Relaciones Públicas en la última década surgieron a raíz de que de la Federación Interamericana de Relaciones Públicas tomó conciencia de que uno de los mayores problemas que afrontaban las Relaciones Públicas era el hecho de que las universidades carecían de una orientación precisa acerca de los contenidos que debían ocluirse en los programas para la formación de profesionales en esta área.

Estas investigaciones fueron realizadas por la Organización Internacional del Trabajo (OIT) a solicitud de la Comisión Interamericana para la enseñanza de las Relaciones Públicas (CIPERP). El método empleado en estos estudios es el de análisis ocupacional. Si bien tiene como inconveniente su antigüedad, veinte años, sin embargo por la seriedad con que fue realizado y por la naturaleza de sus características, sus conclusiones representan un fiel reflejo acerca de cómo se desenvuelve y lleva acabo la profesión de Relaciones Públicas ${ }^{1}$.

Para los propósitos de nuestra investigación, las conclusiones de las investigaciones realizadas por la OIT, para la CIPERP, en sus distintas fases son de alta utilidad en base a los siguientes logros:

Llegar a identificar cinco funciones básicas del profesional de Relaciones Publicas las cuales nos permiten obtener un punto de partida para establecer un aspecto del

\footnotetext{
${ }^{1}$ La Organización Internacional del Trabajo fue establecida por el Tratado de Versalles de 1919, Parte XIII. Después de la segunda guerra mundial, su Constitución fue enmendada para convertirla en organismo especializado de la ONU. Su finalidad consiste en la mejora de la situación de los trabajadores, mediante la adopción de convenios y recomendaciones sobre condiciones laborales. Es característica de la OIT. la composición de su órgano principal, la Conferencia Internacional del Trabajo, que se integra por dos representantes de cada gobierno un representante de los trabajadores y un representante de los empresarios de cada país. La OIT. no se conforma con la adopción de convenios y recomendaciones, sino que también trata de la aplicación de sus normas mediante el estudio de informes enviados por los gobiernos, el envío de comisiones de visita y una labor de vigilancia de la legislación nacional mediante comisiones especiales supervisoras.
} 
perfil del profesional. Se trata del perfil de este profesional en términos de los conocimientos y las destrezas que lo deben caracterizar, y por lo tanto en aquellas que debe prepararse y capacitarse.

Estas cinco funciones básicas son:

1.- Asesoría.

2.- Investigación.

3.- Planificación.

4.- Ejecución. (comunicación)

5.- Evaluación ${ }^{2}$.

Una segunda fase de la investigación nos permite obtener una ampliación y explicación de estos términos funciones> indicándonos qué es lo que sugiere cada una de las cinco funciones básicas, a través del desglose de éstas en diversas funciones especificas. Los resultados son los siguientes ${ }^{3}$ :

No FUNCIONES BÁSICAS SUB-NO FUNCIONES ESPECÍFICAS

1 ASESORÍA

1.1. Sugiere políticas de Relaciones Industriales.

1.2. Sugiere políticas de Mercadología.

1.3. Sugiere políticas Publicitarias.

1.4. Sugiere políticas Rclacionísticas.

1.5. Sugiere actitudes para el tratamiento al público.

2 INVESTIGACIÓN

\footnotetext{
${ }^{2}$ LÓPEZ LÓPEZ, Humberto (1971): La enseñanza de las Relaciones Públicas. Comisión Interamericana para la Enseñanza de las Relaciones Públicas. Medellín (Colombia). Pág. 51.

${ }^{3}$ LÓPEZ LÓPEZ, Humberto (1971): La enseñanza de las Relaciones Públicas. Comisión Interamericana para la Enseñanza de las Relaciones Públicas. Medellín (Colombia). Págs. 51-53.
} 
2.1. Propicia encuestas de opinión pública y analiza sus resultados.

2.2. Analiza archivos de prensa.

2.3. Analiza archivos de correspondencia.

2.4. Realiza entrevistas con Dirigentes de Opinión.

2.5. Define los públicos de su empresa.

2.6. Analiza los públicos de su empresa.

2.7. Propicia y analiza resultados de encuestas de lectorabilidad y lectoría.

2.8. Realiza análisis de Hemerografía.

2.9. Propicia y analiza encuestas de Audiencia.

3 PLANEACIÓN

3.1. Elabora presupuestos para Relaciones Públicas

3.2. Hace estudios de tiempo.

3.3. Elabora un programa y lo experimenta.

3.4. Selecciona personal para ejecutar el programa.

3.5. Presenta y explica el programa.

4.1. Elabora boletines de Prensa.

4.2. Procura, reportajes en los Medios.

4.3. Organiza y dirige Conferencias de Prensa.

4.4. Organiza y dirige Ruedas de Prensa.

4.5. Elabora y dirige las Publicaciones Periódicas de la Empresa.

4.6. Eiafyora y dirige Campañas institucionales por Cine, Radio, Prensa y T.V.

4.7. Elabora y distribuye folletos, libros, desplegables, 
afiches.

4.8. Elabora carteleras, exposiciones, muestras de gestiones, etc.

4.9. Dicta Conferencias.

4.10. Dirige Mesas Redondas, Sesiones de grupo.

4.11. Organiza y coordina visitas a las instalaciones de la empresa y/o viajes.

4.12. Organiza Actos Culturales.

4.13. Organiza Congresos, Conferencias y Seminarios.

4.14. Redacta discursos y menajes.

4.15. Organiza eventos especiales, tales como: Inauguraciones de Instalaciones, Aniversarios de la Empresa, etc.

4.16. Facilita instrumentos de persuasión a otros funcionarios de la empresa.

4.17. Organiza y atiende Actos Sociales.

4.18. Dirige sistemas de Comunicación Interna al personal.

4.19. Toma parte en Sesiones de Grupo.

4.20. Utiliza ayudas audiovisuales.

4.21. Dirige el Ceremonial en actos en que csic es exigido.

4.22. Aplica principios de Relaciones Humanas.

4.23. Toma parle en Corniles de la Empresa.

4.24. Representa a la Empresa en actos diversos.

4.25. Visita a líderes de opinión, empresarios, accionistas, proveedores, distribuidores y consumidores.

4.26. Visita los Medios.

4.27. Sostiene entrevistas y contactos telefónicos, y atiende y resuelve consultas.

4.28. Atiende y responde correspondencia. 
4.29. Facilita información sobre la empresa.

4.30. Recibe sugerencias sobre la empresa.

4.31. Facilita entrevistas con otros directivos de la empresa.

4.32. Dirige el personal de su dependencia.

4.33. Procura la integración de servicios al público.

4.34. Dirige los archivos de prensa, fotografía, el archivo cinematográfico, el de cimas magnetofónicas, el de clichés y planchas litográficas, el de cintas fonópticas y el de textos de documentales.

4.35. Dirige el kárdex o listado de periodistas, personas y entidades de interés para la empresa.

4.36. Detecta inquietudes y fallas en todas las áreas empresariales que puedan afectar al público.

\section{EVALUACIÓN}

5.1. Realiza las mismas tareas consignadas en Investigación.

Veamos ahora el cuadro sinóptico sobre las funciones analizadas del Jefe de Relaciones Públicas. 
REVISTA DE LA SEECI.

Ugalde Fernández, Juan José (1999): Las investigaciones de la Comisión

Interamericana para la enseñanza de las Relaciones Públicas. No 3. Marzo.

Año III. Páginas: 112-140

ISSN: 1576-3420 DOI: http://dx.doi.org/10.15198/seeci.1999.3.112-140

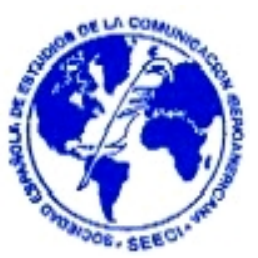

CUadro: JEFE DE RELACIONES PÚBLICAS Y ANÁLISIS DE SUS FUNCIONES

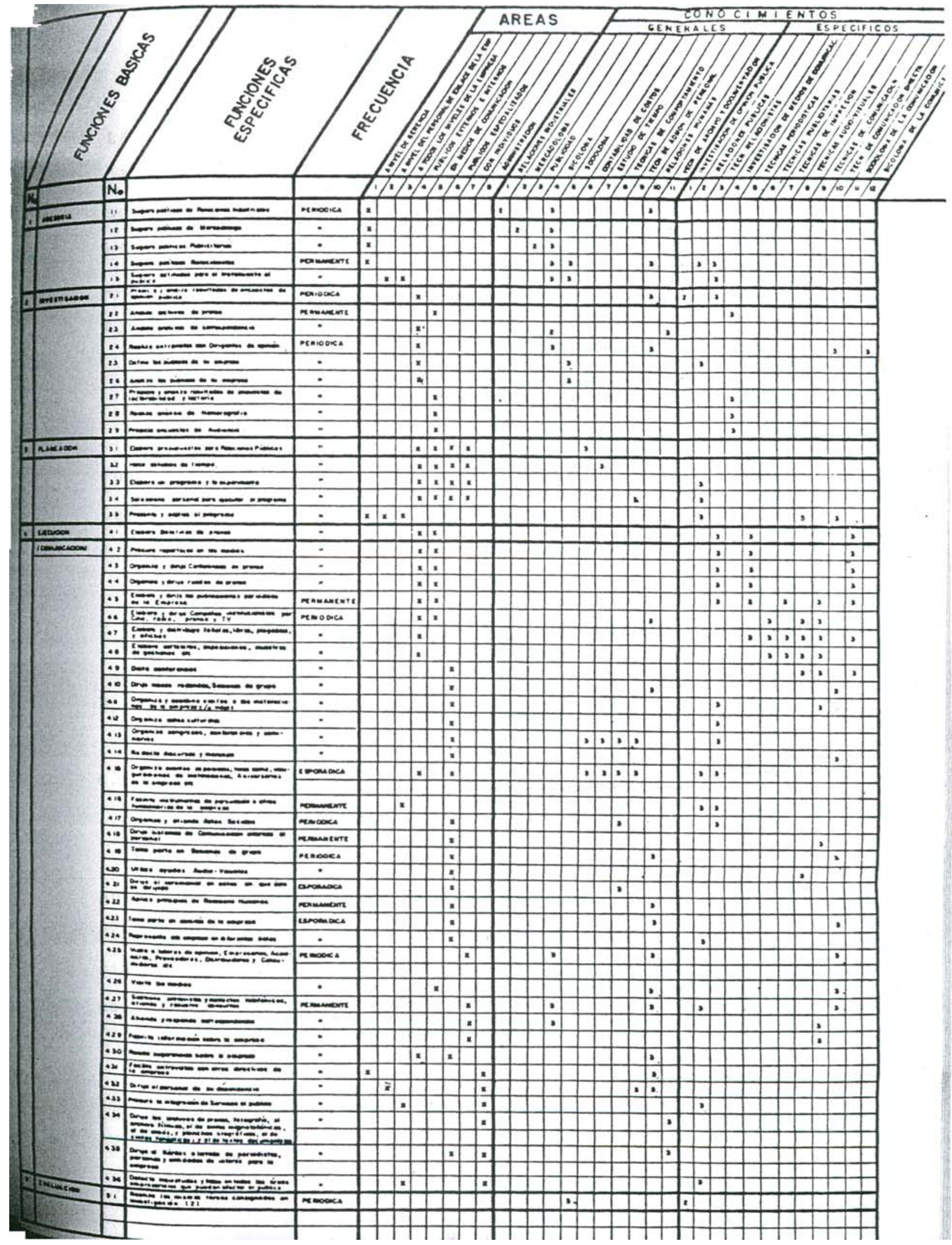


La tercera investigación representada en el cuadro de la anterior página, representa la frecuencia con que se presenta cada una de las funciones específicas y el nivel que ocupan dentro de la jerarquía de mando de las empresas, permitiéndonos obtener datos más exactos y ajustados a la realidad de las funciones del profesional.

A continuación desarrollamos estas funciones:

\subsection{Función básica: Asesoría}

Contempla cinco funciones específicas:

1.- Sugiere políticas de Relaciones Industriales (f. periódica a nivel de gerencia).

2.- Sugiere políticas de mercadología. (f. periódica a nivel de gerencia).

3.- Sugiere políticas publicitarias, (f. periódica a nivel de gerencia).

4.- Sugiere actitudes para el tratamiento del público. (f. permanente a todos los niveles de la empresa).

Análisis de la función básica: Asesoría.

$1,2,3$ y 4 son funciones referidas a la formulación, proposición y dirección de políticas respecto a áreas de la comunicación.

Tres de ellas son periódicas y una es permanente (políticas relacionísticas). Además de pertenecer las cuatro al área de la comunicación, tienen la característica en común de operar a nivel de gerencia.

La función $n^{0} 5$, que sugiere actitudes para el tratamiento del público, podría ser interpretada como políticas para el tratamiento del público, ya que parece razonable entender que el tipo de actitudes que una empresa o institución desea y establece para el tratamiento de su público debe ser formulado y dirigido por una política a nivel gerencial. Sobre todo, si está clasificada en el cuadro $n^{g} 1$, como una función permanente que regula un aspecto muy importante de las comunicaciones con el público. 
Conclusiones acerca de la función básica Asesoría.

1.- Una actividad a nivel gerencial.

2.- Una actividad que se centra y abarca en amplias áreas de la comunicación. $3^{9}$ Una actividad que consiste en formular y proponer políticas comunicacionales. $4^{9}$ Una actividad que en ocasiones es un instrumento del Marketing. Derivaciones de la función básica Asesoría para la configuración del perfil del profesional de Relaciones Públicas.

3.- Que es un comunicador con amplia formación en las distintas áreas de la comunicación, fundamentalmente en relaciones industriales, publicidad, mercadología y tratamiento con el público. Que, no obstante ser un profesional con amplios conocimientos de mercadología, es primeramente un comunicador. Que es un profesional a nivel de dirección, por lo tanto, capacitado para formular, proponer y dirigir políticas comunicacionales.

4.- Que, en consecuencia con lo anterior, es un profesional con conocimientos de administración de empresas y de personal.

\subsection{Función básica: Investigación}

Contempla nueve funciones específicas:

1.- Propicia y analiza resultados de encuestas de opinión pública, (f. periódica a públicos externos e internos).

2.- Analiza archivos de empresa, (f. periódica a públicos externos e internos).

3.- Analiza archivos de correspondencia. (f. permanente a públicos externos e internos).

4.- Realiza entrevistas con dirigentes de opinión. (f. periódica a públicos externos e internos).

5.- Define los públicos de su empresa. (públicos externos internos) .

6.- Analiza los públicos de su empresa. (f. periódica a públicos externos e internos).

7.- Propicia y analiza resultados de encuestas de lectorabilidad y lectoría, (f. periódica en medios de comunicación-, 
8.- Realiza análisis de hemerografía. (f. periódica en medios de comunicación).

9.- Propicia encuestas de audiencia. (f. periódica en medios de comunicación).

Análisis de la función básica: Investigación.

1 y 4 son funciones referidas a la investigación de la Opinión Pública, lo que implica también el análisis y utilización de resultados.

2, 7, 8 y 9 son funciones del área de la investigación de medios de comunicación.

5 y 6 son funciones referidas a la investigación sociológica lógica de públicos que pueden, o no, estar englobadas del marketing.

Conclusiones acerca de la fusión básica: Investigación.

1.- Investigación de la Opinión Pública, de de medios de comunicación y de públicos.

2.- Estas tres funciones de investigación se realizan en el área de públicos externos e internos $(2,3,4,5$ y 6$)$ y en medios de comunicación $(1,7,8$ y 9$)$.

Esta función básica de Investigación, nos sugiere de nuestro profesional:

1.- Que su actividad se manifiesta en base a los conocimientos que le proporcionan las técnicas de investigación sociológicas, sicológicas y mercadológicas acerca de la estratificación de públicos, de las corrientes de Opinión Pública y de la audiencia, alcance y eficacia de los medios de comunicación.

2.- Que es un profesional que conoce las técnicas de investigación mencionadas, en términos de saber lo que puede esperar de cada una de ellas, que sabe analizar y utilizar los resultados que estas le facilitan pero, no es personalmente: un investigador, ya que estas técnicas son solamente aplicadas por sociólogos, sicólogos o técnicos en Marketing.

\subsection{Función básica: Planificación}

Contempla cinco funciones específicas:

1.- Elabora presupuestos para Relaciones Públicas. (f. periódica a públicos externos e 
internos, medios de comunicación, públicos especializados, con individuos)

2.- Realiza estudios de tiempos. (f. periódica a públicos externos e internos, medios de comunicación, públicos especializados con individuos).

3.- Elabora un programa y lo experimenta, (f. periódica a públicos externos e internos, medios de comunicación públicos especializados, con individuos),

4.- Selecciono personal para ejecutar el programa. (f. periódica a públicos externos e internos, medios de comunicación, públicos especializados, con individuos.

5.- Presenta y explica el programa, (f. periódica a nivel de gerencia, a nivel de personal de enlace de le empresa, a todos los niveles de la empresa).

Análisis de la función básica: Planificación.

1 , es una función referida al estudio de los costes de producción y de aplicación de las Relaciones Públicas. 2, 3, 4 y 5 son funciones referidas a la creación de ideas, utilización de recursos humanos y técnicos y a su organización en un programa fundamentado y detallado, para su proposición a quien corresponda.

$1,2,3,4$ y 5 son funciones periódicas referidas fundamentalmente al director de Relaciones Públicas.

Conclusiones acerca de la función básica: Planificación.

1.- Es esta una de las funciones principales y exclusivas del director de Relaciones Públicas. La Planificación de programas no es un tema para ejecutar a niveles inferiores de la profesión.

2.- Es ésta una función que involucra a la economía finanzas y objetivos empresariales o institucionales.

Esta función básica de Planificación, nos sugiere acerca de nuestro profesional:

1.- Que es un profesional experto y máximo responsable en la formulación de estrategias y programas para públicos internos y externos.

2.- Que es un profesional que opera con conocimiento y coordinación de las políticas económicas, financieras y presupuestarias de la empresa o institución. 
3.- Que es un profesional preparado en el área de relaciones inversión-rentabilidad en materia de comunicación.

\subsection{Función básica: Ejecución (Comunicación)}

Contempla treinta y seis funciones específicas:

1.- Elabora boletines de prensa, (f. periódica a públicos externos e internos en medios de comunicación).

2.- Facilita reportajes a los medios. (f. periódica a públicos externos e internos en medios de comunicación).

3.- Organiza y dirige ruedas de prensa. (f. periódica a públicos externos e internos en medios de comunicación).

4.- Organiza y dirige conferencias de prensa, (f. periódica a públicos externos e internos en medios de comunicación).

5.- Elabora y dirige las publicaciones periódicas en la empresa. (f. permanente a públicos externos e internos en medios de comunicación).

6.- Elabora y dirige campañas institucionales para cine, radio, prensa y televisión, (f. permanente).

7.- Elabora y distribuye folletos, libros, plegables y afiches- (f. periódica, públicos externos e internos).

8.- Elabora carteleras, exposiciones, muestras de gestiones, etc, (f. periódica a públicos externos e internos).

9.- Dicta conferencias, (f. periódica).

10.- Dirige mesas redondas, sesiones de grupo, (f. periódica a públicos especializados).

11.- Organiza y coordina visitas a las instalaciones de la empresa y/o viajes, (f. periódica a públicos especializados).

12.- Organiza actos culturales (f. periódica a públicos especializados).

13.- Organiza congresos, conferencias y seminarios. (f. periódica a públicos especializados). 
14.- Redacta discursos sajes (f. periódica a públicos especializados),

15.- Organiza eventos especiales, tales como, inauguraciones, aniversarios de la empresa, etc. (f. esporádica).

16.- Facilita argumentos para persuadir, a otros compañeros de la empresa, (f. permanente).

17.- Organiza y atiende actos sociales. (f. periódica a públicos especializados).

18.- Dirige las comunicaciones internas al personal. (f. permanente a públicos especializados).

19.- Toma parte en sesiones de grupo. (f. periódica a públicos especializados).

20.- Facilita ayudas audiovisuales. (f. periódica a públicos especializados).

21.- Dirige el ceremonial en los actos en que se requiere (f. esporádica a públicos especializados).

22.- Aplica técnicas de Relaciones humanas. (f. permanente a públicos especializados).

23.- Toma parte en las reuniones de los comités de la empresa. (f. esporádica a públicos especializados).

24.- Representa a la empresa en actos diversos. (f. esporádica a públicos especializados).

25.- Visita a líderes de opinión, empresarios, accionistas, proveedores, distribuidores, consumidores, etc. (f. periódica con individuos).

26.- Visita a los medios. (f. periódica en medios de comunicación).

27.- Celebra entrevistas personales por teléfono. (f. permanente con individuos

28.- Atiende y responde correspondencia. (f. permanente con individuos).

29.- Facilita información sobre la empresa, (f. permanente con individuos).

30.- Recibe sugerencias sobre la empresa. (F. permanente a públicos externos, internos y especializados).

31.- Facilita entrevistas con otros directivos de la empresa. (f. permanente a nivel de gerencia y administración).

32.- Dirige el personal de su dependencia, (f. permanente con el personal dedicado a Relaciones Públicas). 
33.- Procura la integración de servicio al público. (f. permanente a todos los niveles de la empresa).

34.- Dirige los archivos de prensa, fotografía, el archivo fílmico, el de cintas magnetofónicas, el de clisés y planchas litográficas, el de cintas fonópticas y el de textos documentales, (f. permanente de administración).

35.- Dirige el kárdex o listado de periodistas, personas y entidades de interés para la empresa, (f. permanente).

36.- Detecta inquietudes y faltas en todas las áreas empresariales que puedan afectar al público, (f. permanente a todos los niveles de la empresa).

Análisis de la función básica: Ejecución (Comunicación).

24,27 y 28 son funciones que se basan en la sicología.

13 y 15 son funciones referidas a contabilidad de costos y a estudios de tiempo.

$13,15,17$ y 21 son funciones referidas a técnicas de comportamiento.

13,15 y 32 son funciones referidas a técnicas de Administración de personal.

$6,10,19,22,23,25,26,27,30,31$ y 32 son funciones referidas a las relaciones humanas.

34 y 35 son funciones referidas a técnicas de archivo y documentación.

$15,17,24,33$ y 36 son funciones referidas a las Relaciones Públicas.

$1,2,3,4,5,11,12,15,16$ y 17 son funciones referidas a las técnicas relacionistas

$1,2,3,4$ y 5 son funciones referidas a técnicas de periodismo.

6,7 y 8 son funciones referidas a técnicas publicitarias.

5,7 y 8 son funciones referidas a técnicas de impresión.

$6,7,8,9$ y 20 son funciones referidas a técnicas audiovisuales.

$5,6,7,8,9,11,18,26$ y 27 son funciones referidas a técnicas de comunicación.

$10,14,19,23,25,26$ y 27 son funciones referidas a técnicas de comunicación directa.

$1,2,3,4,5,7$ y 9 son funciones respaldadas por la sociología de la comunicación.

Función básica: Ejecución (Comunicación). 
Distribución de las 36 funciones en las 14 técnicas:

11 se fundamentan en las relaciones humanas.

11 se fundamentan en técnicas relacionísticas.

9 se fundamentan en técnicas de comunicación.

7 se fundamentan en técnicas de comunicación directa.

6 se fundamentan en técnicas de Relaciones Públicas.

6 se fundamentan en técnicas audiovisuales.

5 se fundamentan en técnicas periodísticas.

4 se fundamentan en técnicas de comportamiento.

3 se fundamentan en técnicas de administración de personal.

3 se fundamentan en técnicas publicitarias.

3 se fundamentan en técnicas de impresión.

2 se fundamentan en contabilidad.

2 se fundamentan en estudios de tiempos.

2 se fundamentan en técnicas de archivo y documentación.

De estas 35 funciones, 3 se apoyan la sicología y 7 en la sociología de la comunicación.

Conclusiones acerca de la función básica: Ejecución. (Comunicación).

Primer grupo.- Sería el grupo central en torno al cual se aglutinan las técnicas más cercanas o propias de las Relaciones públicas, e incluyen 6 de las 14 técnicas:

- Relaciones Públicas.

- Relaciones humanas.

- Técnicas relacionistas.

- Técnicas de comunicación directa.

- Técnicas de comportamiento.

- Administración de personal.

Segundo grupo.- Está constituido por las técnicas de comunicación, incluyendo de las 14 técnicas. 
- Técnicas de comunicación.

- Técnicas periodísticas.

- Técnicas publicitarias.

- Técnicas de impresión.

- Técnicas audiovisuales.

Tercer grupo.-

- Contabilidad de costos.

- Estudios de tiempo.

- Técnicas de archivo y documentación.

Esta función básica de Ejecución (Comunicación) nos sugiere acerca de nuestro profesional:

1.- Que su actividad se basa en su preparación y capacidad para canalizar las relaciones humanas empresariales o institucionales, internas o externas.

2.- Que su actividad tiene como finalidad el control de conductas a través de técnicas de relaciones y de comunicaciones interpersonales, grupales y masivas.

3.- Que, en gran medida actúa apoyado en la psico-sociología, respaldos necesarios para su actividad.

4.- Que su actividad está enmarcada en un medio económico en el que tiempo y costos son variables que inciden significativamente en el rendimiento y eficacia de su acción.

5.- Que tiene un nivel de preparación en técnicas de archivo y documentación de materiales propios de su actividad comunicacional. (películas, fotos, clichés, listados de periodistas, etc.).

Función básica: Evaluación.

Contempla las mismas funciones específicas indicadas para la función básica de la investigación, lo que significa que estarían incidiendo de la misma manera que esta función para la configuración del perfil del profesional de las Relaciones Públicas. 


\section{Posiciones de diversos autores y profesionales de las Relaciones Públicas en cuanto al perfil de la profesión}

Estudiaremos las opiniones de algunos de los "clásicos", con incidencia en la cultura norteamericana y europea.

Scott M. Cutlip y Allen H. Center- La definición del Public Relations News, incluye los tres objetivos fundamentales del profesional de las Relaciones Públicas ${ }^{4}$.

1.- Establecer y evaluar la Opinión Pública.

2.- Asesorar a los directivos sobre la forma de manejar la Opinión Pública en sus diversos aspectos.

3.- Emplear los medios de comunicación adecuados para influir en la Opinión Pública.

Estos mismos autores destacan ${ }^{5}$ que

"Los profesionales no deben caer en el error de creerse a sí mismos los responsables por derecho propio de las relaciones entre una corporación y sus diversos sectores de público. Prácticamente ello significaría la arrogación de las funciones más importantes de la administración. En suma, no se contrata a los especialistas en Relaciones públicas en calidad de gerentes".

Desde un punto de vista funcional, los directivos contratan al relacionador público ${ }^{6}$ :

1.- "Para facilitar y asegurar la recepción de una corriente de opiniones representativas emitidas por los diversos sectores de

\footnotetext{
${ }^{4}$ CUTLIP, S. M. y CENTER, A. H. (1985): Effective Public Relations. Englewood Cliffs, N.J. Prentice Hall, revised 6 th. ed. 1985. Págs. 18-19.

${ }^{5}$ CUTLIP, S. M. y CENTER, A. H. (1985): Effective Public Relations. Englewood Cliffs, N.J. Prentice Hall, revised 6 th. ed. 1985. Pág. 21.

${ }^{6}$ CUTLIP, S. M. y CENTER, A. H. (1985): Effective Public Relations. Englewood Cliffs, N.J. Prentice Hall, revised 6 th. ed. 1985. Pág. 21.
} 
público de la organización y mantener de este modo una política y un método de operar compatibles con las diversas necesidades y puntos de vista de esos públicos.

2.- Para aconsejar a la gerencia sobre los medios a emplear en la orientación de esa política y de esos métodos operativos, al objeto de obtener el máximo apoyo público respecto a lo que a su juicio reviste importancia fundamental para todos los interesados.

3.- Para interpretar amplia y favorablemente la política y los métodos operativos de la organización".

Más adelante continúan explicando este papel de puente que cumple el relacionador público entre la empresa y sus clientes. De esta manera, el profesional, en su papel de agente de enlace e intérprete, contribuye de forma vital al problema de adaptar la institución al clima de la evolución social de forma que se sirvan indistintamente y en la medida de lo posible, tanto los intereses públicos como los privados.

No obstante, la confusión que existe con respecto al concepto y aplicaciones de las Relaciones Públicas, lo que si esta claro para Cutlip y Center (1985) es que estas tienen como fin "ganar y conservar las opiniones favorables de los públicos respecto a una institución o industria".

En su interés de delimitar las funciones del profesional de Relaciones Públicas, estos mismos autores nos puntualizan su papel en relación con la administración y dirección de las instituciones, en los siguientes términos ${ }^{7}$ :

"Podría decirse que la función de las Relaciones Públicas es la de no dirigir ni administrar una institución. El profesional no está a cargo de todas las relaciones de la institución y los conocimientos adquiridos no le capacitan para ser un experto en todo... los

\footnotetext{
7 CUTLIP, S. M. y CENTER, A. H. (1985): Effective Public Relations. Englewood Cliffs, N.J. Prentice Hall, revised 6 th. ed. 1985.
} Págs. 249-250. 
directivos son los que determinan la política básica. Los especialistas de Relaciones Públicas colaboran con ellos para lograr un impacto eficaz en la Opinión Pública y también es posible que se les convoque para opinar sobre otros asuntos. Las Relaciones Públicas es una función administrativa $y$, por lo tanto, los que la practican deben conocer el papel del cuerpo administrativo en la organización... el cuerpo administrativo desempeña las funciones fundamentales: estas abarcan al personal, la planificación, las Relaciones Públicas y la publicidad".

Continuando con el tema de los conocimientos y destrezas científicas y técnicas, estos autores ${ }^{8}$ insisten en lo que sin duda, parece ser el centro y clave de la formación de un profesional de las Relaciones Públicas cuando afirman:

"Las Relaciones Públicas exigen la habilidad y preparación de verdaderos científicos de la Opinión Pública en mayor grado que los productores de información, los artistas u otros técnicos rigurosamente especializados".

Además apuntan otro rasgo del perfil del profesional del cual dependerá su desarrollo y éxito en su trabajo, al afirmar:

"Una de las causas principales que conducen al fracaso, es la incapacidad para aprender el negocio. No la incapacidad para aprender la técnica de las Relaciones Públicas, sino para comprender de forma concisa y profunda tanto si se trata de un pequeño comerciante, de un gran industrial de una institución benéfica o de una universidad. El primer paso es ganarse la confianza de los directivos y ello sólo pueden lograrlo los profesionales cuya experiencia les permita comprender los problemas de la dirección....

\footnotetext{
${ }^{8}$ CUTLIP, S. M. y CENTER, A. H. (1985): Effective Public Relations. Englewood Cliffs, N.J. Prentice Hall, revised 6 th. ed. 1985.
} Pág. 383. 
La mayoría de los asesores y directores de Relaciones Públicas de hoy día fueron originalmente periodistas, publicistas y anunciantes... el típico director de Relaciones Públicas es un hombre de unos cuarenta y seis años de edad, que asistió a la universidad y se graduó en comercio o periodismo, que ha practicado uno de los dos, posee habilidad especial para tratar a la gente, expresa sus pensamientos con claridad y sencillez, ya sea verbalmente o por escrito; conoce la administración de la empresa, dedica una gran parte de su tiempo a representar a su organización ante diversos públicos y cuenta con ayudantes que poseen una educación universitaria más completa que la suya propia...El especialista del futuro habrá de poseer un conocimiento profundo de las ciencias sociales, con cuya ayuda se convertirá en un verdadero informador y no simplemente en un divulgador. Asimismo, habrá de hacer uso de conocimientos de sicología social, sociología industrial y evaluación de opiniones, para interpretar a los gerentes las diferentes opiniones representativas. La práctica contemporánea requiere el talento de un especialista capacitado, no el de un publicista".

También estos autores se refieren a otros "NO" 9 en relación con nuestro profesional, citando un informe de la revista Business Week (Semana de negocios), los cuales se concretan en siete puntos:

1.- El profesional de Relaciones Públicas tiende a ser excesivamente un "yes man" ( $S I^{\prime}$ a todo).

2.- Los altos directivos encuentran que a menudo el profesional de las Relaciones Públicas no queda bien en las reuniones de alto nivel. Hay cierta timidez o meticulosidad excesiva.

\footnotetext{
${ }_{9}^{9}$ CUTLIP, S. M. y CENTER, A. H. (1985): Effective Public Relations. Englewood Cliffs, N.J. Prentice Hall, revised 6 th. ed. 1985.
} Págs. 391-392. 
3.- El profesional de Relaciones Públicas tiene una inadecuada formación, en general, sobre negocios y economía. De toda la gente de la plana mayor de la empresa, es el que menos comprende la filosofía de los negocios o los problemas básicos de distribución, de la producción y de la investigación.

4.- Más concretamente, el profesional de las Relaciones Públicas no comprende a menudo el trabajo de la compañía para la cual actúa. Puede tener un buen conocimiento de la distribución, de las relaciones laborales, etc, pero frecuentemente, aparece absolutamente en blanco, cuando se trata de investigación, desarrollo o finanzas.

5.- El profesional de las Relaciones Públicas, frecuentemente no se preocupa de los problemas inmediatos de la compañía. "Mi consejero de Relaciones Públicas me dice organizar un cierto tipo de visitas a mi fábrica cuando mi problema inmediato es concluir un convenio laboral" "Por qué no me presenta un plan para ayudar a resolverlo", dice el presidente de una compañía.

6.- En nuestra compañía, el departamento se conoce como el Teatro de la Opera. Tiene más 'primas donnas' que el Metropolitan, fue uno de los comentarios.

7.- El profesional de las Relaciones Públicas es demasiado susceptible en lo que se refiere a si se le ha solicitado o no su consejo. "Mi hombre de Relaciones Públicas no parece darse cuenta completa de que su consejo es uno de los muchos consejos o asesoramientos que tengo que tener en cuenta al tomar una decisión".

Noam Chomsky ${ }^{10}$.- Este autor cita a Bernays, haciendo hincapié en el carácter persuasivo de la función del profesional, transcribiéndonos las siguientes afirmaciones de éste:

"...La industria de las Relaciones Públicas que no ha cesado de florecer desde que su poder de adoctrinamiento quedó puesto de manifiesto eficazmente durante la primera guerra mundial. Las Relaciones públicas no son más que la producción en masa de buenas maneras personales y buenos hábitos morales. La primera

\footnotetext{
${ }^{10}$ CHOMSKY, Noam (1984): La segunda guerra fría. Crítica. Barcelona. Pág. 91.
} 
figura en el campo de las Relaciones Públicas, Edward Bernays, ha dicho cosas muy interesantes sobre estas cuestiones. Los dirigentes... de los principales grupos organizados... con la ayuda de los técnicos... que se han especializado en la utilización de los canales de comunicación, han sido capaces de llevar a. cabo... científicamente lo que hemos dado en llamar 'instrumentación del consenso', explicaba en los Annals of the American Academy of Political and Sciencie... La frase instrumentación del consenso, continua Bernays, significa pura y simplemente la aplicación de principios científicos y prácticas comprobadas a la tarea de conseguir que la gente de su apoyo a las ideas y a los programas... La instrumentación del consenso es la esencia misma del proceso democrático, la libertad de convencer y proponer... A menudo un dirigente no puede esperar a que el pueblo llegue a tener siquiera una comprensión general de tal o cual cosa... los dirigentes democráticos deben desempeñar su función en la instrumentación del consenso en torno a objetivos y valores socialmente positivos. Una vez más, son los empresarios y sus representantes en el gobierno quienes juzgarán en la práctica qué es socialmente positivo".

Fernando Vera Carranza.- Óscar Fernández Balzano ${ }^{11}$ cita a Fernando Vera Carranza, quien en pocas palabras nos ofrece un perfil de nuestro profesional en términos globales afirmando lo siguiente:

"... El especialista en Relaciones Públicas no es un simple informante, ni tampoco el mágico intérprete que saca de apuros al industrial $o$ al economista extranjero que encuentra problemas en nuestro medio. Es ante todo y esencialmente un hombre culto, de conocimientos

\footnotetext{
${ }^{11}$ FERNÁNDEZ BALZANO, Óscar (1973): Manual de Relaciones Públicas y Relaciones Humanas. Para la enseñanza media, básica y superior. Estudio Argentino de Relaciones Públicas. Buenos Aires.
} 
humanísticos clásicos y modernos. Es así que estudia filosofía, historia, arte, sociología, estética literaria, ceremonial, comunicaciones, y al mismo tiempo profundiza la economía y la política económica. Como sicólogo práctico pero sutil, sabe conquistar amigos para la empresa, que se convertirán en clientes y propagandistas".

Rafael Torres Padial.- Este autor, cuyo programa de Relaciones Públicas se imparte en varias universidades norteamericanas, también centra la labor del profesional de Relaciones Públicas en torno al manejo de la Opinión Pública, con lo que nuevamente vemos que se refuerza la postura de la mayoría de los autores consultados, acerca de que el perfil de nuestro profesional incluye sólidos conocimientos de esta materia. Al respecto, cita Rafael Torres Padia ${ }^{12}$ :

"Una empresa, como un estado o un partido político, tienen que contar con una corriente de Opinión Pública favorable. Un producto no se extenderá si la empresa tiene una imagen social baja o deleznable. La corriente de opinión es fundamental y básica, y canalizarla es un deber primordial del Jefe o Director de Relaciones Públicas".

Primer encuentro Iberoamericano de Profesores de Relaciones Públicas.- Este Congreso celebrado en Castelldefels del 20 al 25 de Mayo de 1985, y en el que tuve el honor de participar con una comunicación, estableció unas conclusiones, que se adjuntan, definiendo las funciones básicas de la profesión de Relaciones Públicas.

El Primer encuentro Iberoamericano de Profesores de Relaciones Públicas, acordó que las citadas conclusiones se recomendarán aplicar en todos los países que integran la Confederación Iberoamericana de Relaciones Públicas, haciéndolas llegar a los órganos gubernamentales pertinentes de cada país.

\footnotetext{
${ }^{12}$ TORRES PADIAL, Rafael (1972): El asesor Publicitario. Cuadernos de Publicidad. Madrid. Pág. 11.
} 
Su línea, muy acorde con la establecida por la OIT, estableció las tres funciones básicas:

- Diagnosticar (Investigación).

- Planificación y ejecución de los programas

- Control y evaluación de resultados.

Insistiendo de una forma especial en la formación teórico-práctica de nuestro profesional.

Nadie cuestiona en nuestro tiempo la necesidad de un conocimiento total de la empresa y entidad, y también aparece en estas conclusiones (no ocurre así en en informe de la OIT.) que su conducta ha de ser ética.

Pasamos a enumerar el informe final que se elaboró tras el I Encuentro Iberoamericano de Profesores de Relaciones Públicas a modo de desiderándum sobre la función docente de los contenidos anteriormente expresados en todas las áreas de la función publirrelacionística.

\section{3. $1^{\text {er }}$ enCUENTRO IBEROAMERICANO DE PROFESORES DE RELACIONES PÚBLICAS AUSPICIADO POR LA CONFEDERACIÓN IBEROAMERICANA DE RELACIONES PÚBLICAS (CIRP)}

\subsection{Conclusiones del Encuentro:}

A fin de legitimar el poder de la Organización para conseguir niveles óptimos de Mercado ante sus Públicos y la Sociedad, se definen como FUNCIONES BÁSICAS de la profesión de Relaciones Públicas:

1. ANALIZAR las tendencias de la Organización y de sus Públicos en el ámbito de su coyuntura, en función de los intereses legítimos de ambos. 
2. DIAGNOSTICAR las situaciones que configuran las tendencias de ambas partes.

3. PRONOSTICAR las posibles consecuencias.

4. ASESORAR a los Directivos o responsables de la Organización sobre las Políticas Generales de Acción.

5. PLANIFICAR E IMPLEMENTAR Programas de Comunicación.

6. CONTROLAR el desarrollo de los programas y EVALUAR sus resultados.

Para desarrollar estas funciones el Profesional de Relaciones Públicas tiene que:

1. Conocer la Teoría de las Relaciones Públicas y ciencias afines.

2. Saber diagnosticar y pronosticar la relación Organización/ Públicos.

3. Saber crear alternativas estratégicas y técnicas para evitar y solucionar problemas específicos de Relaciones Públicas.

4. Saber negociar sus puntos de vista con los Directivos.

5. Saber ejercer sus funciones administrativas.

6. Tener una conducta ética.

En consecuencia el "Curriculum" debe:

1. Informar sobre Teoría de Relaciones Públicas y sobre ciencias afines.

2. Capacitar para diagnosticar, pronosticar y tomar decisiones sobre alternativas de solución a problemas de Relaciones Públicas.

3. Capacitar para utilizar técnicas de información y comunicación.

4. Capacitar en administración empresarial.

5. Capacitar para la negociación de sus puntos de vista profesionales.

6. Concienciar acerca de las realidades socio-cultural-económico-político- morales de la profesión y de la sociedad.

Paro lo cual el "Curriculum " debe estar basado en: 
1. La filosofía de la Institución docente y de la comunidad de Relaciones Publicas.

2. La legislación vigente sobre la enseñanza de Relaciones Públicas.

3. La relación de materias según un criterio racional, teórico y práctico.

4. La coordinación interdisciplinaria de las materias.

5. La utilización de técnicas de metodología activa, principalmente los métodos de casos.

6. Las prácticas (Stage) en Organizaciones.

7. Los encuentros entre y de profesores y estudiantes.

8. El perfeccionamiento permanente de los profesores.

El "Curriculum" será ampliado y adaptado a la realidad de cada país.

Relación de Materias:

Materias Básicas:

1. Teoría de Relaciones Públicas.

2. Tecnología de Relaciones Públicas.

3. Teoría de la Información y de la Comunicación.

4. Técnicas de Información y de Comunicación (oral, no verbal, escrita, medios de información de masas).

5. Teoría e Investigación de Opinión Pública.

6. Planificación.

7. Odontología.

8. Legislación e Historia de las Relaciones Públicas.

Materias Accesorias:

1. Teoría de Administración.

2. Teoría de las Organizaciones. 
3. Teoría política.

4. Marketing.

5. Sociología.

6. Economía.

7. Psicología (General, Social y Organizacional).

8. Derecho (Público, Privado, Mercantil y Teoría del Estado).

9. Antropología Cultural (Culturas y lingüística).

10. Filosofía (Ética, Estética, y Epistemología).

11. Comunicación Comparada (medios de información en el espacio y en el tiempo).

12. Estructura socio-económica-política del país.

13. Estadística.

14. Idioma nacional.

Materias Optativas:

1. Informática.

2. Idiomas Extranjeros.

3. Lógica.

4. Historia del Pensamiento (Ideología).

5. Ciencia de la Historia.

6. Geografía Económica.

\subsection{Acuerdo final}

El I Encuentro Iberoamericano de Profesores de Relaciones Públicas, acuerda adoptar las presentes conclusiones y recomendar la aplicación de lo allí contenido en todos los países que integran la Confederación Iberoamericana de Relaciones Públicas, haciéndolas llegar a los órganos gubernamentales pertinentes de cada país. 


\section{Bibliografía}

BARQUERO CABRERO, José Daniel y BARQUERO CABRERO, Mario (1996). El libro de oro de las Relaciones Públicas. Gestión 2000. Barcelona.

CHOMSKY, Noam (1984). La segunda guerra fría. Crítica. Barcelona.

CUTLIP, Scott M. y CENTER, Allen H. (1985). Effective Public Relations. Englewood Cliffs, N.J. Prentice Hall, revised 6 th. ed. 1985.

FERNÁNDEZ BALZANO, Óscar (1973). Manual de Relaciones Públicas y Relaciones Humanas. Para la enseñanza media, básica y superior. Estudio Argentino de Relaciones Públicas. Buenos Aires.

LÓPEZ LÓPEZ, Humberto (1971). La enseñanza de las Relaciones Públicas. Comisión Interamericana para la Enseñanza de las Relaciones Públicas. Medellín (Colombia).

MONZÓN ARRIBAS, Cándido (1996). Opinión Pública y Política. Tecnos. Madrid.

SOLANO FLETA, Luis (1995). Fundamentos de las Relaciones Públicas. Síntesis. Madrid.

SOLANO FLETA, Luis (1999. Tratado de las Relaciones Públicas. Gestión 2000. Barcelona.

TORRES PADIAL, Rafael (1972). El asesor Publicitario. Cuadernos de Publicidad. Madrid. 